\title{
哏翟
}

ISSN: 2474-3542 Journal homepage: http://journal.calaijol.org

\section{Linked Data in a Nutshell: A Starter Kit of Selected Annotated Bibliography and Resources for Academic Librarians: Part Two}

\author{
Weiling Liu
}

\begin{abstract}
:
It has been a decade since Tim Berners-Lee coined Linked Data in 2006. More and more Linked Data datasets have been made available for information retrieval on the Web. It is essential for librarians, especially academic librarians, to keep up with the state of Linked Data. There is so much information about Linked Data that one may wonder where to begin when they want to join the Linked Data community. With this in mind, the author compiled this annotated bibliography as a starter kit. Due to the many resources available, this list focuses on literature in English only and of specific projects, case studies, research studies, and tools that may be helpful to academic librarians, in addition to the overview of Linked Data concept and the current state of Linked Data evolution and adoption.
\end{abstract}

To cite this article:

Liu, W. (2018). Linked Data in a nutshell: a starter kit of selected annotated bibliography and resources for academic librarians: part two. International Journal of Librarianship, 3(1), 54-66. doi: https://doi.org/10.23974/ijol.2018.vol3.1.51

To submit your article to this journal:

Go to http://ojs.calaijol.org/index.php/ijol/about/submissions 


\title{
Linked Data in a Nutshell: A Starter Kit of Selected Annotated Bibliography and Resources for Academic Librarians: Part Two
}

\author{
Weiling Liu \\ University of Louisville, Louisville, Kentucky, USA
}

\begin{abstract}
It has been a decade since Tim Berners-Lee coined Linked Data in 2006. More and more Linked Data datasets have been made available for information retrieval on the Web. It is essential for librarians, especially academic librarians, to keep up with the state of Linked Data. There is so much information about Linked Data that one may wonder where to begin when they want to join the Linked Data community. With this in mind, the author compiled this annotated bibliography as a starter kit. Due to the many resources available, this list focuses on literature in English only and of specific projects, case studies, research studies, and tools that may be helpful to academic librarians, in addition to the overview of Linked Data concept and the current state of Linked Data evolution and adoption.
\end{abstract}

Keywords: Linked Data, Linked Open Data, Library Linked Data, Semantic Web, Academic Library, Bibliography

\section{INTRODUCTION}

Resources for Part One are selected with a focus on Linked Data concepts, current state, and case studies of Linked Data evolution and adoption in a university setting. Resources for Part Two are selected with a focus on research studies of Linked Data vocabularies, Library Linked Data, novel approaches and tools used to address issues and challenges related to Linked Data implementation. The publication dates of the resources are within the past five years from 2017. Literature covered in Part Two include 14 journal articles, one book, two conference papers, one committee document, one book chapter, one research project report, and a few websites. The bibliographies are grouped into five sections: 1) Studies or Research Projects on Linked Data;2) Novel Approaches and Tools; 3) Vocabularies; 4) Library Linked Data, and 5) Websites. This starter kit is by no means a comprehensive list; the author attempts to list some examples of projects or research studies that illustrate the use of Linked Data, and solutions or approaches that deal with some specific challenges encountered in the Linked Data publication and consumption. Like Part One, Part Two also lists some related websites. The bibliographies are in 
ascending chronological order by year then by author for easy reading and also listed in the References.

\section{STUDIES AND RESEARCH PROJECTS}

This section includes some recent publications of research studies on Linked Data, revealing the impact of Linked Data, current state of adoption, and problems or issues to be resolved.

Moulaison, H. L., \& Million, A. J. (2014). The disruptive qualities of Linked Data in the library environment: Analysis and recommendations. Cataloging \& Classification Quarterly, 52(4), 367-387. doi:10.1080/01639374.2014.880981

In this article, the authors evaluate the disruptive qualities of Linked Data and the implication for libraries based on Christensen's disruptive technologies theory. They conclude that Linked Data may play a role in improving bibliographic library technology, but there is not much evidence that indicates it will emerge as a disruptive technology in the library environment. They also recommend that it is still premature at this time to use Linked Data to encode library data, but it is one of many solutions that should be explored in a systematic and controlled way.

Niknia, M., \& Mirtaheri, S. L. (2015). Mapping a decade of Linked Data progress through coword analysis. Webology, 12(2). Retrieved from http://www.webology.org/2015/v12n2/a141.pdf

The authors conducted a study on the evolution of Linked Data based on 717 Scopus articles and their associated 19977 keywords published between the 1970s and 2014. The study uses co-word analysis and hierarchical cluster analysis along with strategic diagrams and network analysis to map and visualize the progress of Linked Data as a research topic. The results show that studies on linked data began in the 1970s and commonly associated with computer science. The explosion of publication is observed since 2008 with the focus shifted from technology to the Semantic Web. The results also show that the USA has published the highest number of articles. In conclusion, the authors suggest that studies on user experience may become important after computing-related issues are solved.

Hallo, M., Luján-Mora, S., Maté, A., \& Trujillo, J. (2016). Current state of Linked Data in digital libraries. Journal of Information Science, 42(2), 117-127.

doi:10.1177/0165551515594729

The authors present a study about the current uses of Linked Data in digital libraries (LDDL) concerning the benefits, vocabularies, and ontologies used, problems and future trends. The study was conducted using an adapted methodology for literature review based on information extracted from a group of selected publications and five national libraries websites worldwide. 
The paper includes the description of each selected library's Linked Data project and discussions of the findings along with a comparison table of features of selected libraries. It also offers suggestions for ways in which libraries can contribute to the Semantic Web. For example, the preservation of Linked Datasets and the adoption of 5-star Linked Open Data standards.

Arlitsch, K. (2017). Semantic web identity of academic libraries. Journal of Library Administration, 57(3), 346-358. doi:10.1080/01930826.2017.1288970

This article is a summary of part of the research for a recent doctoral dissertation written by Arlitsch. The study is about the Semantic Web Identity (SWI) of ARL (Association of Research Libraries) Libraries and other academic organizations. The research hypothesis is that "certain proprietary and open Semantic Web knowledge bases must be actively engaged so that an organization can be optimally recognized and understood as an entity by Google" (Arlitsch, 2017, p. 350). Arlitsch argues that the implication of SWI goes far beyond the simple display of a Knowledge Graph Card in Google search engine results pages. SWI may help strengthen the chances for funding for a research center or student enrollment, faculty recruitment, etc. for an academic institution.

\section{NOVEL APPROCHES AND TOOLS}

To tackle some issues of Linked Data, researchers have developed tools or novel approaches. This section collected some publications on Linked Data quality assessment and management. Although the literature included here focuses on Linked Data in general, it is helpful for librarians to know about these tools or approaches that have been experimented.

Sequeda, J. F., \& Miranker, D. P. (2013). Ultrawrap: SPARQL execution on relational data. Web Semantics: Science, Services and Agents on the World Wide Web, 22, 19-39. doi:10.1016/j.websem.2013.08.002

The authors postulate that existing legacy relational databases already include the algorithms and optimizations required to execute SPARQL queries on RDF representation of the legacy relational data. To resolve the contradiction among two previous studies, the authors built Ultrawrap, a system which not only can detail an RDF graph representation of the relational data based on the W3C RDB2RDF Direct Mapping standard but also translate the relational schema and accompany SQL constraints into an OWL (the Web Ontology Language) ontology. In this article, the authors explore the hypothesis with Ultrawrap, and the results suggest that "effective wrappers will be those that are designed to complement the optimizer of the target database." (Sequeda, \& Miranker, 2013, p. 19).

Ristoski, P., Bizer, C., \& Paulheim, H. (2015). Mining the web of Linked Data with RapidMiner. Web Semantics: Science, Services and Agents on the World Wide Web, 35, 142-151. doi:10.1016/j.websem.2015.06.004 
This article presents the full functionality of the RaidMiner Linked Open Data extension. This extension is a part of RapidMiner, a data mining and analysis platform. The RaidMiner Linked Open Data extension allows "operators" to access Linked Open Data in RapidMiner and use it in sophisticated data analysis workflow without the need for expert knowledge in SPARQL or RDF. These "operators" are fundamental building blocks in RapidMiner, and each of them performs a specific action on data, such as data import, data linking, and feature generation. Through an example with statistical data from the World Bank, the article illustrates how relevant datasets can be discovered on the fly, and overlapping data found in different datasets can be integrated. The extension is available for free download from the RapidMiner marketplace under the AGPL license at https://marketplace.rapidi.com/UpdateServer/faces/product_details.xhtml?productId=rmx_lod

Debattista, J., Auer, Sö., \& Lange, C. (2016). Luzzu—A methodology and framework for Linked Data quality assessment. J. Data and Information Quality, 8(1), 4:1-4:32. doi:10.1145/2992786

The article defines a conceptual methodology for assessing Linked Data quality based on the data quality lifecycle. Through a thorough discussion, the article illustrates the possibilities of Luzzu, a modular, open-source framework, for assessing Linked Data quality regarding scalability, extensibility, interoperability, and customizability. The article concludes that although there are some limitations, Luzzu framework allows users (both Linked Data publisher and consumer) to analyze data quality from a single visual entry point easily. This project is only the first step in the long-term research agenda, and the next step is to examine techniques for incremental quality assessment.

With descriptions and a table, the article also compares Luzzu against nine other available Linked Data quality assessment frameworks from a different angle than that was used in a previous investigation done by other researchers in 2015.

Dunsire, G., Fritz, D., \& Fritz, R. (2016). Instructions, interfaces, and interoperable data: The RIMMF experience with RDA. Paper presented at IFLA WLIC 2016. Retrieved from http://library.ifla.org/1324/1/093-dunsire-en.pdf

This article presents a case study of a software tool, RIMMF (Resource Description and Access (RDA) in Many Metadata Formats). RIMMF, developed by TMQ, Inc., bridges the gap between professional cataloguers and the technologies underpinning legacy (i.e., MARC Machine-Readable Cataloging) and modern (i.e., Linked Data) metadata. RIMMF is based on FRBR (Functional Requirements for Bibliographic Records) and RDA terminology, but it can export data for Linked Data applications in RDF (Resource Description Framework). This function allows catalogers to produce well-formed Linked Data without the new technical skills and also help to improve their understanding of the complexities involved. RIMMF is still a prototype and training tool. However, it has been extensively tested "'in the field' in a series of 
hackathons for RDA data, known as 'jane-athons' from an initial focus on the works of Jane Austen" (Dunsire, Fritz \& Fritz, 2016, p.7). This article also includes an overview of the evolution of bibliographic rules and standards such as RDA and FRBR - a conceptual bibliographic model published by IFLA (International Federation of Library Associations and Institutions).

Kalou, A. K., Koutsomitropoulos, D. A., \& Solomou, G. D. (2016). Combining the best of both worlds: A semantic web book mashup as a Linked Data service over CMS infrastructure. Journal of Library Metadata, 16(3-4), 0-0. doi:10.1080/19386389.2016.1258897

This article proposes a proof-of-concept Web-based application, Books@HPClab - a semantic mashup, which combines RESTful services, semantic technologies, and CMS (Content Management System). The article illustrates how personalized book recommendations can be generated from various Web sources and made available as Linked Data via this mashup. The authors also demonstrate how commercial metadata can be enriched with bibliographic information via Linked Data principles. It also shows the potentials of the application for use in digital libraries, open educational repositories, online learning systems, and online course providers due to its enclosed automated Web services. The article also includes the evaluation of the application's performance to see how well it can match user search needs (effectiveness) and how quickly the results are retrieved (efficiency).

\section{Rietveld, L. (2016). Publishing and consuming Linked Data: Optimizing for the unknown. IOS Press.}

This dissertation presents new novel approaches for tackling some technical problems related to publishing and consuming Linked Data. The issues include many areas, from data reuse to hosting solutions, finding, navigating and using Linked Datasets. There is also the lack of resources and methods for scientists to evaluate their work on Linked Data in general. It first gives an overview of the problems from the perspectives of three Linked Data stakeholders: the provider, developer and scientist, the proposed solutions, and contents of each chapter. It then provides detailed descriptions of the problems, associated background and related works as well as proposed solutions. As the author concludes, these solutions improve the state of the art for Linked Data publishers, developers and scientists. They are a step towards a web of Linked Data that is more accessible and technically scalable (Rietveld, 2016, p. 143).

The paper is very well organized. Each chapter discussing a solution including an introduction and conclusion to recap the problem and summarize the answers. Some implementations are described in a step-by-step manner. Readers may visit the sites to learn more about the approaches discussed in the paper: LOD Laundromat (http://lodlaundromat.org/), SampLD (https://github.com/Data2Semantics/GraphSampling), YASGUI (http://doc.yasgui.org/), and LOD Lab (http://lodlaundromat.org/lodlab/). 
Lee, Y. (2017). A life-cycle workflow architecture for Linked Data. In Proceedings of the 2017 International Conference on Machine Learning and Soft Computing (pp. 117-121). New York, NY, USA: ACM. doi:10.1145/3036290.3036302

This article describes phase one of a research plan aiming to address the need for scalable data management solutions that can efficiently store, index and query native RDF data. The article provides a systematic overview of the proposed novel Linked Data life-cycle workflow architecture. The architecture includes four components: "Acquisition System," "Ontology Learning Method," "RDF Store \& MDH*" and "Analysis System." The article describes each component of the architecture, such as storage, indexing, query processing and application approaches of Linked Data and how related issues are addressed. Through the descriptions and visual diagrams, readers get a better idea of the whole life-cycle of Linked Data and associated problems.

\section{VOCABULARIES}

"The promise of the Web of Data is not only to enable client applications to discover new data sources by following RDF links at run-time but also to help them to integrate data from these sources" (Heath \& Bizer, 2011, p. 24). Vocabularies or ontologies are a crucial component in Linked Data. They are the bridges between data sources in data integrations. In this section, readers will find publications about projects or studies with a focus on this aspect.

Oh, S. G., Yi, M., \& Jang, W. (2015). Deploying linked open vocabulary (LOV) to enhance library linked data. Journal of Information Science Theory and Practice, 3(2), 6-15. doi:10.1633/JISTaP.2015.3.2.1

This article discusses the potential role of Linked Open Vocabularies (LOV), a portal for RDFS (Resource Description Framework Schema) and OWL (Web Ontology Language), in improving the implementation of Linked Data in the library community. The article evaluates the LOV system based on the criteria developed by a previous study. It also gives a comparative analysis with some significant open data repositories; the report proposes a few approaches in which LOV can be used to improve linking as well as access to open Linked Datasets. The suggestions include the use of LOV for building an open and specialized Library Linked Data repository, and for classifying, mapping, and analyzing data for Big Data archiving.

The URLs to the LOV and evaluated open data repositories are listed below.

- LOV system: http://lov.okfn.org/dataset/lov/

- Open Data Repositories

○ Datahub.io: http://datahub.io/

- LODStats: http://stats.lod2.eu/

- Datacatalogs.org: http://datacatalogs.org/ - (Now it is dataportals.org)

- European Union Open Data Portal: https://open-data.europa.eu/en/data/ 
Binding, C., \& Tudhope, D. (2016). Improving interoperability using vocabulary linked data. International Journal on Digital Libraries, 17(1), 5-21. doi:10.1007/s00799-015-0166-y

The authors discuss various aspects of vocabulary mapping through a case study for the ARIADNE project, based on the Getty Art and Architecture Thesaurus as a hub. The study results indicate the enhanced opportunities for interactive vocabulary mapping and cross-searching. In this pilot experiment, the authors also demonstrate the potential use of the vocabulary mapping tool developed for the project to assist cross-search over archaeological datasets from different countries. The authors point out that the problems of expressing high-quality mappings between data and a reference vocabulary are related to the issues involved in mapping between vocabularies as Linked Data.

Radio, E., \& Hanrath, S. (2016). Measuring the impact and effectiveness of transitioning to a Linked Data vocabulary. Journal of Library Metadata, 16(2), 80-94.

doi:10.1080/19386389.2016.1215734

Through a test case transitioning the metadata of an institutional repository to OCLC's FAST (Faceted Application of Subject Terminology), this article attempts to measure the accuracy of mapping pre-existing terms to a controlled vocabulary and its impact on usage and resource discovery. The authors note that the study results indicate a need for increased attention and participation to identify areas of under- or misrepresentation in Linked Data vocabularies. They also note that one should not expect any immediate increase in usage: "There is no explicit promise that exposing resources as Linked Data will result in such an event, but not participating in Linked Data activities may prove to be a missed opportunity" (Radio \& Hanrath, 2016, p. 92).

\section{LIBRARY LINKED DATA}

The library community began its work on exploring ways to replace MARC around the same time when Tim Berners-Lee proposed Linked Data principle in 2006. Influenced by Linked Data principles, the library leading organizations have launched Linked Data initiatives and collaborative projects. In this section, readers will find selected recent reports, documents, and publications that explore the use of Library Linked Data (or Linked Library Data) or demonstrate the development of Linked Data activities in the library community, particularly, those from the major players such as the Library of Congress and OCLC.

Konstantinou, N., Houssos, N., \& Manta, A. (2014). Exposing bibliographic information as linked open data using standards-based mappings: Methodology and results. Procedia Social and Behavioral Sciences, 147(1), 260-267. doi:10.1016/j.sbspro.2014.07.169

This article describes an approach that uses international standards, such as Library of Congress Linked Data Service for generating and publishing Linked Data in the case of digital repositories that contain bibliographic information. It presents not only the methodology used in 
implementation but also various available options. It discusses several benefits (semantic annotation, query simplification, increased discoverability, and reusability) and challenges associated with the approach (multidisciplinary, technology barrier, concept mismatch, and exceptions to the general rule). The article concludes that the investment in semantics pays off in a long run although Linked Open Data generation requires some initial resource investment in person-hours.

Godby, C.J., \& Denenberg. R. (2015). Common ground: Exploring compatibilities between the Linked Data models of the Library of Congress and OCLC. Retrieved from https://www.oclc.org/content/dam/research/publications/2015/oclcresearch-loc-linkeddata-2015.pdf

This white paper was jointly released by OCLC and the Library of Congress (LC). The article compares the Linked Data initiatives at both institutions, explaining the differences between LC's BIBFRAME and OCLC's model which is based on Schema.org. It includes a figure which visualizes the differences between the two models as well as an overview of the recent activities of both institutions since 2013 .

Phipps, J., Dunsire, G., \& Hillmann, D. (2015). Building a platform to manage RDA vocabularies and data for an international, Linked Data world. Journal of Library Metadata, 15(3-4), 252-264. doi:10.1080/19386389.2015.1099990

The authors describe the Open Metadata Registry (OMR) on which RDA (Resource Description and Access) is built as well as the evolution of the current RDA Registry including the rationale for decisions, directions, and ongoing development.

Tharani, K. (2015). Linked Data in libraries: A case study of harvesting and sharing bibliographic metadata with BIBFRAME. Information Technology \& Libraries, 34(1), $5-19$.

In this article, Tharani presents a case study in which BIBFRAME is used for harvesting and sharing bibliographic metadata on the Web for the libraries. It gives a step-by-step explanation of the implementation as well as an overview of BIBFRAME and a detailed explanation of Linked Data. In this project, technology and format used for data preparation are very common, for example, MySQL for storing data and XSLT (Extensible Stylesheet Language Transformations) scripts for transforming data. One exception is the generation of RDF triples, for which the specific programming effort is described as complex. Through this case study, the article demonstrates BIBFRAME's potential in making digital libraries more accessible, and the challenges BIBFRAME is facing. Tharani points out that "[a]lthough initially introduced as a replacement for MARC, BIBFRAME is far from being an either-or proposition given the MARC legacy" (2015, p. 16). 
Library of Congress, Acquisitions \& Bibliographic Access Directorate (ABA). (2016, June 16). BIBFRAME pilot (Phase One-Sept. 8, 2015 - March 31, 2016): Report and assessment. Retrieved from https://www.loc.gov/bibframe/docs/pdf/bibframe-pilot-phase1analysis.pdf

This ABA report assesses the Library of Congress' first BIBFRAME (Bibliographic Framework Initiative) pilot. The report includes two parts that were prepared respectively by NDMSO (the Network Development \& MARC Standards Office) and COIN (the Cooperative \& Instructional Programs Division) of ABA. This pilot is phase one based on BIBFRAME 1.0 environment with a focus on the input of data and impact on catalogers. Part one of the report reviews the preparation and training, including training, pilot environment, workflow, lessons learned and best practices. Part two reviews the pilot system based on some questions that were articulated before the start of the pilot. BIBFRAME 2.0 is already available, and phase two of the pilot is currently in progress according to the report. However, this report seemed to be current on BIBFRAME's website (http://www.loc.gov/bibframe/docs/) when this annotated bibliography was in writing.

Colye, K. (2016). Bibliographic description and the Semantic Web. In FRBR, Before and After: A Look at Our Bibliographic Models (pp.137-156). Retrieved from http://kcoyle.net/beforeAndAfter/c10-978-0-8389-1364-2.pdf

This chapter demonstrates through specific projects how the conceptual model of FRBR is used in recent RDF vocabularies developed for library data and in bibliographic models designed outside of the library. Projects discussed here include those that have transformed the entity-relation model of FRBR, such as FRBRer, RDA in RDF, BIBFRAME, and the projects that use alternation of FRBR, for example, FRBRcore, FRBRoo's Object-Oriented Model, $<$ indecs $>$ Event-Oriented Model, and Fabio FRBR-Aligned Bibliographic Ontology. In summary, the chapter highlights that FRBRcore and BIBFRAME are the projects that show promise as vocabularies for the open Web. In this chapter, readers will see how FRBR is related to BIBFRAME, RDA in RDF, and differences among the projects.

McCallum, S. H. (2017). BIBFRAME development. JLIS.it, 8(3), 71-85. http://doi.org/10.4403/jlis.it-12415

In the article, McCallum discusses the development of Linked Data in the Library of Congress (LC) over the past decade. The report examines the evolution through five areas: using RDF, LC Linked Data Service, Developing BIBFRAME (Bibliographic Framework Initiative), models, and exploring.

Readers will learn from this article not only the overview of development history but also how Linked Data principles align with LC's goals recommended in On the Record: Report of the Library of Congress Working Group on the Future of Bibliographic Control in 2008. Through the concrete examples, readers will see the differences between current formats and Linked Data 
principles. Readers will also see how BIBFRAME is related to FRBR (Functional Requirements for Bibliographic Records), RDA (Resource Description and Access), and MARC (Machinereadable Cataloging).

Baxmeyer, J., Coyle, K., Dylan, J., Han, M. J., Folsom, S., Schreur, P., \& Thompson, T. (2017, June). Linked Data infrastructure models: Areas of focus for PCC strategies. Retrieved from https://www.loc.gov/aba/pcc/documents/LinkedDataInfrastructureModels.pdf

This white paper is prepared for the Library of Congress' PCC (Program Cooperative Cataloging) Linked Data Advisory Committee. The paper gives an overview of PPC's four components: BIBCO (Monographic Bibliographic Record Cooperative Program), CONSER (Cooperative Online Serials Program), NACO (Name Authority Cooperative Program), and SACO (Subject Authority Cooperative Program). It describes the known challenges with Linked Data in the library environment and outlines some high-level requirements. In the appendix of this paper, it also lists some grant-funded projects and library system vendors who are active with Linked Data. Although it is a document prepared for PCC's internal committee, it gives general readers an overview of issues associated with Linked Data in the library community.

\section{WEBSITES}

This section aims to provide readers with a quick reference list of selected website resources that are related to the focus of Part Two. The author idenitfies them here in this section for readers' convenience because they are good for keeping up-to-date on Linked Data development and helpful for implementation.

Creative Commons (https://creativecommons.org)

Creative Commons is a global nonprofit organization. It enables sharing and reuse of creativity and knowledge. The combination of its solutions and users is "a vast and growing digital commons, a pool of content that can be copied, distributed, edited, remixed, and built upon, all within the boundaries of copyright law" (Creative Commons, 2018). The licenses and tools provided by Creative Commons "were designed specifically to work with the web, which makes content that is offered under their terms easy to search for, discover, and use" (Creative Commons, 2018).

International Semantic Web Conference (ISWC)

ISWC is a series of international academic conferences forum, for the Semantic Web/ Linked Data Community. Since 2002, each year scientists, industry specialists, and practitioners come from all over the world to discuss "the future of practical, scalable, user-friendly, and game changing solutions" (ISWC, 2018, March 26). The conferences are usually held in different countries, and there is no central website. However, Wikipedia lists all the links to the annual 
meetings. The conference proceedings are published in the Lecture Notes in Computer Science by Springer-Verlag.

\author{
Linked Library Data Interest Group (LITA / ALCTS) \\ (http://www.ala.org/lita/about/igs/linked/lit-iglld)
}

Originated in 2011, the Linked Library Data Interest Group is a joint interest group of LITA (Library and Information Technology Association) and ALCTS (Association for Library Collections \& Technical Services). It is entirely self-initiated and not appointed by its parent associations or ALA (American Library Association). This group attempts to provide an avenue for librarians to discuss issues related to Library Linked Data.

Open Knowledge International (https://okfn.org/)

Open Knowledge International is a global non-profit organization. It focuses on realizing open data's value to society. It helps civil society groups access and use data to take action on social problems. It supports many initiatives, such as CKAN (an open source data management solution) and Global Open Data Index. One of its projects, Open Definition, defines Open Bibliographic Data (https://opendefinition.org/bibliographic/).

RightsStatements.org (http://rightsstatements.org/en/about.html)

RightsStatements.org, a joint initiative of Europeana and DPLA (the Digital Public Library of America), provides 12 standardized rights statements specifically for the needs of cultural heritage institutions and online digital aggregation platforms. As stated on the About page, these statements provide a minimum, baseline standard for any digital aggregator that adopts the RightsStatements.org standard as well as Europeana and organizations contributing to the DPLA. These rights statements established by RightsStatements.org are ready for use by institutions to communicate the copyright and re-use status of digital cultural heritage objects to the public. The rights statements are also made available as linked data.

\title{
SUMMARY
}

The author hopes that Part Two has provided readers more resources that may lead to a more indepth understanding of Linked Data, especially issues and challenges encountered in its implementation as well as its benefits in the library, archive, and museum community. As stated in "Library Linked Data Incubator Group Final Report," the ownership of library data is complicated and data with unclear and untested rights issues can hinder their release as Open Data (Baker et al., 2011). The resources selected in this kit were a side product of the author's six-month sabbatical project about an ideal assistant system or application for library resource searching in 2017. Given the complexity of data, limited time, and original focus on informationseeking behaviors and information discovery, the kit still lacks some resources such as those 
dealing with rights issues. Hopefully, the website resources about rights and licenses for Linked Data included here are useful to readers.

\section{References}

Arlitsch, K. (2017). Semantic web identity of academic libraries. Journal of Library Administration, 57(3), 346-358. doi:10.1080/01930826.2017.1288970

Baker, T., Bermès, E., Coyle, K., Dunsire, G., Isaac, A., Murray, P., . . Zeng, M. (2011). Library Linked Data Incubator Group final report. Retrieved from W3C Incubator. World Wide Web Consortium website: https://www.w3.org/2005/Incubator/lld/XGR-lld20111025/

Baxmeyer, J., Coyle, K., Dylan, J., Han, M. J., Folsom, S., Schreur, P., \& Thompson, T. (2017, June). Linked Data infrastructure models: Areas of focus for PCC strategies. Retrieved from https://www.loc.gov/aba/pcc/documents/LinkedDataInfrastructureModels.pdf

Binding, C., \& Tudhope, D. (2016). Improving interoperability using vocabulary linked data. International Journal on Digital Libraries, 17(1), 5-21. doi:10.1007/s00799-015-0166-y

Colye, K. (2016). Bibliographic description and the Semantic Web. In FRBR, Before and After: A Look at Our Bibliographic Models (pp.137-156). Retrieved from http://kcoyle.net/beforeAndAfter/c10-978-0-8389-1364-2.pdf

Debattista, J., Auer, Sö., \& Lange, C. (2016). Luzzu-A methodology and framework for Linked Data quality assessment. J. Data and Information Quality, 8(1), 4:1-4:32. doi:10.1145/2992786

Dunsire, G., Fritz, D., \& Fritz, R. (2016). Instructions, interfaces, and interoperable data: The RIMMF experience with RDA. Paper presented at IFLA WLIC 2016. Retrieved from http://library.ifla.org/1324/1/093-dunsire-en.pdf

Creative Commons. (2018, March 7). Frequently asked questions. Retrieved from https://creativecommons.org/faq/\#what-is-creative-commons-and-what-do-you-do

Godby, C.J., \& Denenberg. R. (2015). Common ground: Exploring compatibilities between the Linked Data models of the Library of Congress and OCLC. Retrieved from https:/www.oclc.org/content/dam/research/publications/2015/oclcresearch-loc-linked-data2015.pdf

Hallo, M., Luján-Mora, S., Maté, A., \& Trujillo, J. (2016). Current state of Linked Data in digital libraries. Journal of Information Science, 42(2), 117-127. doi:10.1177/0165551515594729

Heath, T., \& Bizer, C. (2011). Linked Data: Evolving the web into a global data space. 1st ed. Synthesis lectures on the semantic web: Theory and technology, 1:1. San Rafael, CA: Morgan \& Claypool. Retrieved from http://linkeddatabook.com/editions/1.0

Wikipedia. (2018, March 26). International semantic web conference (ISWC). Retrieved from https://en.wikipedia.org/w/index.php?title=International_Semantic_Web_Conference\&oldid $=832489256$

Kalou, A. K., Koutsomitropoulos, D. A., \& Solomou, G. D. (2016). Combining the best of both worlds: A semantic web book mashup as a Linked Data service over CMS infrastructure. Journal of Library Metadata, 16(3-4), 0-0. doi:10.1080/19386389.2016.1258897 
Konstantinou, N., Houssos, N., \& Manta, A. (2014). Exposing bibliographic information as linked open data using standards-based mappings: Methodology and results. Procedia Social and Behavioral Sciences, 147(1), 260-267. doi:10.1016/j.sbspro.2014.07.169

Lee, Y. (2017). A life-cycle workflow architecture for Linked Data. In Proceedings of the 2017 International Conference on Machine Learning and Soft Computing (pp. 117-121). New York, NY, USA: ACM. doi:10.1145/3036290.3036302

Library of Congress, Acquisitions \& Bibliographic Access Directorate (ABA). (2016, June 16). BIBFRAME pilot (phase one-Sept. 8, 2015 -March 31, 2016): Report and assessment. Retrieved from https://www.loc.gov/bibframe/docs/pdf/bibframe-pilot-phase1-analysis.pdf

McCallum, S. H. (2017). BIBFRAME development. JLIS.it, 8(3), 71-85. http://doi.org/10.4403/jlis.it-12415

Moulaison, H. L., \& Million, A. J. (2014). The disruptive qualities of Linked Data in the library environment: Analysis and recommendations. Cataloging \& Classification Quarterly, 52(4), 367-387. doi:10.1080/01639374.2014.880981

Niknia, M., \& Mirtaheri, S. L. (2015). Mapping a decade of Linked Data progress through coword analysis. Webology, 12(2). Retrieved from http://www.webology.org/2015/v12n2/a141.pdf

Oh, S. G., Yi, M., \& Jang, W. (2015). Deploying linked open vocabulary (LOV) to enhance library Linked Data. Journal of Information Science Theory and Practice, 3(2), 6-15. doi:10.1633/JISTaP.2015.3.2.1

Phipps, J., Dunsire, G., \& Hillmann, D. (2015). Building a platform to manage RDA vocabularies and data for an international, Linked Data world. Journal of Library Metadata, 15(3-4), 252-264. doi:10.1080/19386389.2015.1099990

Radio, E., \& Hanrath, S. (2016). Measuring the impact and effectiveness of transitioning to a Linked Data vocabulary. Journal of Library Metadata, 16(2), 80-94. doi:10.1080/19386389.2016.1215734

Rietveld, L. (2016). Publishing and Consuming Linked Data: Optimizing for the Unknown. IOS Press.

Ristoski, P., Bizer, C., \& Paulheim, H. (2015). Mining the web of Linked Data with RapidMiner. Web Semantics: Science, Services and Agents on the World Wide Web, 35, 142-151. doi:10.1016/j.websem.2015.06.004

Sequeda, J. F., \& Miranker, D. P. (2013). Ultrawrap: SPARQL execution on relational data. Web Semantics: Science, Services and Agents on the World Wide Web, 22, 19-39. doi:10.1016/j.websem.2013.08.002

Tharani, K. (2015). Linked Data in libraries: A case study of harvesting and sharing bibliographic metadata with BIBFRAME. Information Technology \& Libraries, 34(1), 519.

\section{About the author}

Weiling Liu is Professor and Head of Office of Libraries Technology at University of Louisville, Louisville, Kentucky, USA. 